Proceedings of the National Academy of Sciences: Vol. 109, pp. 19697-19702, 2012

\title{
FoxO is a critical regulator of stem cell maintenance in immortal Hydra
}

\author{
Anna-Marei Boehm', Konstantin Khalturin', Friederike A. Erxleben', Georg Hemmrich', Ulrich C. \\ Klostermeier ${ }^{2}$, Javier A. Lopez-Quintero' ${ }^{1}$, Hans-Heinrich Oberg ${ }^{3}$, Malte Puchert ${ }^{1}$, Philip Rosenstiel ${ }^{2}$, \\ Jörg Wittlieb ${ }^{1}$ and Thomas C. G. Bosch ${ }^{1}$
}

'Zoological Institute, Christian-Albrechts-University, 24098 Kiel, Germany; ${ }^{2}$ Institute of Clinical Molecular Biology (IKMB), University Hospital Schleswig-Holstein, 24105 Kiel, Germany; ${ }^{3}$ Institute of Immunology, University Hospital Schleswig-Holstein, 24105 Kiel, Germany

\section{Rahul Tyagi}

Neuroscience Research Lab, Department of Neurology, Post Graduate Institute of Medical Education and Research, Chandigarh, INDIA

\section{Background}

Immortality has always been an issue of curiosity among scientific community. Hydra's indefinite self renewal capacity of stem cells has attracted natural scientists to seek insights about longevity studies in humans. Three stem cell lineages ectodermal and endodermal epitheliomuscular stem cells and interstitial stem cells contribute to indefinite self renewal capacity of epithelial cells. Moreover, forkhead boxO (foxO) which plays a key role in increased life span in lower creatures like flies and worms has shown to have a strong association of FoxO3a gene in human longevity and maintainence of adult haematopoitic stem cells and neural stem cells. ${ }^{1}$ Recent studies in beetles have shown the effect of fox homologous genes in increased life span. ${ }^{2}$ The above group has studied the expression of transcription factor foxO in the stem cell lineages and illustrated the effect of gain of function and loss of function to confirm the effect of foxO in controlling stem cell function and longevity. Gain of function of FoxO revealed the proliferation of stem cells and conferred stemness whereas silencing of the same resulted in terminal differentiation.

\section{Methodology}

Hydra Vulgeris strain AEP was cultured and transgenic strain was generated through foxO-eGFP and foxO-hairpin transgene to achieve overexpression and knockdown of foxO respectively using actin promoter. FoxO overexpression was analysed in interstitial and nematoblast precursor cells whereas foxo knockdown was analysed in six transgenic lines. Proliferation was detected using 5-bromo-2'-deoxyuridine (BrdU) after $6 \mathrm{~h}$ of exposure. FoxO gain and loss of function was analysed through in-situ hybridization and quantitative real time PCR (qRT-PCR). Phylogenetic analysis were carried out using 31 amino acids of fox domain.

\section{Implications}

This study has established the role of forkhead box proteins in longevity by exploring the immortal characteristics of hydra which shows no sign of aging and reproductive loss. This study has revealed the role of foxO in early evolution of stem cell and in maintaining stem cell renewal capacity. FoxO has role to play in studying aging process and reduction of immunity functions with future implications for regenerative medicine.

doi : $10.5214 /$ ans. 0972.7531 .200107

Reference

1. Willcox BJ, Donlon TA, He Q, et al. FOXO3A genotype is strongly associated with human longevity. Proc Natl Acad Sci UCA. 2008; 105 (37): 13987-13992.

2. Grünwald S, Stellzig J, Adam IV, et al. Longevity in the red flour beetle Tribolium castaneum is enhanced by broccoli and depends on nrf2, jnk-1 and foxo-1 homologous genes. Genes Nutr. 2013. [Epub ahead of print] 